\title{
Efficacy and safety of nimotuzumab with neoadjuvant chemotherapy followed by concurrent chemoradiotherapy for locoregionally advanced nasopharyngeal carcinoma
}

\author{
Fangzheng Wang ${ }^{1,2, *}$, Chuner Jiang ${ }^{3, *}$, Zhimin Ye ${ }^{1,2}$, Quanquan Sun ${ }^{1,2}$, Tongxin Liu ${ }^{1,2}$, \\ Min Xu' ${ }^{4}$, Peng Wu ${ }^{5}$, Kaiyuan Shi ${ }^{6}$, Bin Long7, Aizawa Rihito ${ }^{8}$, Sakamoto Masoto ${ }^{8}$ \\ and Zhenfu Fu' ${ }^{1,2}$ \\ ${ }^{1}$ Department of Radiation Oncology, Zhejiang Cancer Hospital, Zhejiang Hangzhou, People's Republic of China \\ ${ }^{2}$ Zhejiang Key Laboratory of Radiation Oncology, Zhejiang Hangzhou, People's Republic of China \\ ${ }^{3}$ Department of Breast Tumor Surgery, Zhejiang Cancer Hospital, Zhejiang Hangzhou, People's Republic of China \\ 4 Department of Physics, Zhejiang Cancer Hospital, Zhejiang Hangzhou, People's Republic of China \\ ${ }^{5}$ Department of Pathology, Zhejiang Cancer Hospital, Zhejiang Hangzhou, People's Republic of China \\ ${ }^{6}$ Department of Ultrasonography, Zhejiang Cancer Hospital, Zhejiang Hangzhou, People's Republic of, China \\ 7 Department of Nuclear Medicine, Zhejiang Cancer Hospital, Zhejiang Hangzhou, People's Republic of China \\ ${ }^{8}$ Department of Radiology, Japanese Red Cross Fukui Hospital, Fukui, Japan \\ * These authors have contributed equally to this work \\ Correspondence to: Fangzheng Wang, email: wangfz76@126.com \\ Keywords: nasopharyngeal carcinoma, nimotuzumab, neoadjuvant chemotherapy, concurrent chemotherapy, intensity-modu- \\ lated radiotherapy \\ Received: January 28, $2017 \quad$ Accepted: April 11,2017 Published: April 21, 2017
}

Copyright: Wang et al. This is an open-access article distributed under the terms of the Creative Commons Attribution License 3.0 (CC BY 3.0), which permits unrestricted use, distribution, and reproduction in any medium, provided the original author and source are credited.

\section{ABSTRACT}

We assessed the efficacy and safety of nimotuzumab plus neoadjuvant chemotherapy followed by concurrent chemoradiotherapy for Chinese patients with locoregionally advanced nasopharyngeal carcinoma. Clinical data from 210 nonmetastatic nasopharyngeal carcinoma patients diagnosed between May 2008 and April 2014 were retrospectively reviewed. All patients were initially treated with nimotuzumab plus neoadjuvant chemotherapy followed by concurrent chemoradiotherapy. Ninety-five patients received cisplatin-based adjuvant chemotherapy. The median follow-up duration was $\mathbf{4 8}$ months. Locoregional relapse and distant metastases occurred in 16 patients $(16 / 210,7.6 \%)$ and 18 patients $(18 / 210,8.6 \%)$, respectively. The 5-year local recurrence-free survival, regional recurrence-free survival, distant metastases-free survival, progression-free survival, and overall survival rates were $95.6 \%, 94.4 \%, 91.7 \%, 84.0 \%$, and $88.7 \%$, respectively. Univariate analysis revealed that concurrent chemotherapy regimens and clinical stage correlated with overall survival, and that adjuvant chemotherapy, $\mathbf{N}$ stage, clinical stage, and tumor response at the end of treatment were correlated with progression-free survival. In the multivariate analysis, concurrent chemotherapy regimens, clinical stage, and tumor response were important prognosticators. Grade $3 / 4$ leukocytopenia was experienced by 24 patients $(11.4 \%)$, and 6 patients $(2.9 \%)$ developed mild liver damage during the period of neoadjuvant chemotherapy. Grade $3 / 4$ acute mucositis was experienced by 13 patients $(6.2 \%)$, and 12 patients $(5.7 \%)$ experienced grade 3/4 leukocytopenia during the concurrent chemotherapy. The efficacy of nimotuzumab plus neoadjuvant chemotherapy followed by concurrent chemotherapy in locoregionally advanced nasopharyngeal carcinoma patients was encouraging and the toxicities were tolerable. 


\section{INTRODUCTION}

The incidence of nasopharyngeal carcinoma (NPC) is 15 to 50 cases per 100,000 annually in Southern China, Singapore, and Malaysia that vary with age, ethnicity, and geographical origin [1]. Radiotherapy (RT) is the standard treatment for NPC because of the anatomical location and the high radiosensitivity. Patients with locoregionally advanced (LA) NPC at diagnosis account for $60 \%$ to $70 \%$ of all NPC patients [2]. Intensity modulated radiation therapy (IMRT) has been used to improve locoregional control but provides little benefit for survival outcome and prevention of distant failure [3, 4]. According to metaanalyses of randomized studies, combination radiotherapy and chemotherapy reduces the risk of mortality by $18 \%$ and increases 5 -year survival by $4 \%$ to $6 \%$ [5]. Concurrent chemoradiotherapy (CCRT) with or without adjuvant chemotherapy, which provides a benefit in overall survival, has become the standard treatment for LA NPC, although with acute toxicities [6-8]. A previous metaanalysis showed that compared with CCRT alone, addition of neoadjuvant chemotherapy (NAC) to CCRT reduces distant failure in LA NPC patients [9, 10], and another current meta-analysis confirmed that NAC followed by CCRT significantly improved progression-free survival (PFS) and overall survival (OS) [11]. However, the efficacy of the addition of NAC to CCRT in LA NPC patients remains controversial [12-14]. Considering these results, addition of NAC to CCRT has been a promising option for LA NPC patients in the era of IMRT. At present, the incidence of distant failure in LA NPC patients after combined treatment is more than $20 \%$ [15]. Therefore, new and effective regimens with tolerable toxicity for LA NPC are needed.

Overexpression of epidermal growth factor receptor (EGFR) is observed in many different cancers, including gliomas, sarcomas, and head and neck cancers [16]. Moreover, high EGFR expression is associated with poor prognosis $[17,18]$. Several inhibitors of EGFR, such as cetuximab, panitumumab, erlotinib, and gefitinib, have shown favorable results in clinical trials $[19,20]$. Cetuximab, the most commonly used anti-EGFR antibody, combined with radiotherapy (RT), has been shown to improve survival in patients with locoregionally advanced head and neck squamous cell carcinoma (LA HNSCC) [21]. In NPC, cetuximab with concurrent chemoradiotherapy is tolerable and has shown promising advantages for NPC prognosis [22]. However, the relatively high rate of mucositis and acne-like skin rash limit its clinical application [22,23].

Nimotuzumab is a blocking anti-EGFR monoclonal antibody without intrinsic stimulating activity [24]. In the preclinical studies, nimotuzumab demonstrated antiproliferative, proapoptotic, and antiangiogenic activities [25], and nimotuzumab displayed a longer halflife and elevated area under the curve than cetuximab at the same dose level [26]. Nimotuzumab improves quality of life because it rarely causes severe dermatological toxicity, which is the most common adverse event resulting from cetuximab and panitumumab use [27].

Nimotuzumab has marketing approval for the treatment of LA NPC [28, 29]. However, the value of adding nimotuzumab to NAC followed by CCRT in LA NPC patients remains unclear. Therefore, we retrospectively investigated the safety and efficacy of nimotuzumab plus NAC followed by CCRT in LA NPC patients.

\section{RESULTS}

\section{Patient characteristics and completion of treatment}

Between May 2008 and April 2014, the clinical data of 210 newly diagnosed LA NPC patients, who were initially treated with nimotuzumab plus NAC followed by CCRT in the Department of Radiation Oncology, Zhejiang Cancer Hospital (Hangzhou, People's Republic of China), were collected and retrospectively reviewed. Basic characteristics of patients are summarized in Table 1. All patients completed a full course of radical IMRT and received 1 to 4 cycles of NAC, $\geq 1$ cycle of concurrent chemotherapy (CC), and 3 to 17 weeks of nimotuzumab (Table 2). Among these patients, 95 (45.2\%) received adjuvant chemotherapy (AC).

\section{Disease response}

At the end of treatment, complete remission (CR) and partial remission (PR) for lesions of the nasopharynx in 210 LA NPC patients accounted for $83.8 \%$ (176/210) and $16.2 \%(34 / 210)$, respectively. For 189 patients with neck metastatic lymph nodes, CR and PR rates of cervical lymph nodes were $88.9 \%(168 / 189)$ and $11.1 \%(21 / 189)$, respectively.

\section{Rates of local control and survival}

The median follow-up time was 48 months (range, 13-75 months). The estimated 5-year local recurrencefree survival (LRFS), regional recurrence-free survival (RRFS), distant metastasis-free survival (DMFS), progression-free survival (PFS), and overall survival (OS) rates were $95.6 \%, 94.4 \%, 91.7 \%, 84.0 \%$, and $88.7 \%$, respectively (Figure 1). The 5-year OS rates were $100 \%$, $90.1 \%, 91.0 \%$, and $83.8 \%$ for patients with stage $\mathrm{T} 1, \mathrm{~T} 2$, T3, and T4 disease, respectively $(P=0.080)$ (Figure 2A). However, the patients with stage T4 disease had poorer OS rates than those with stage T1-T3 disease $(83.8 \% v s$. 
Table 1: Basic characteristic of 210 LA NPC patients

\begin{tabular}{|l|l|}
\hline Characteristic & $\mathbf{N}(\mathbf{\%})$ \\
\hline Gender & \\
\hline Male & $154(73.3)$ \\
\hline Female & $56(26.7)$ \\
\hline Age (years) & \\
\hline Range & $13-72$ \\
\hline Median & 46 \\
\hline$<50$ & $131(62.4)$ \\
\hline$\geq 50$ & $35(37.6)$ \\
\hline WHO pathology & \\
\hline Type I & $9(4.3)$ \\
\hline Type II & $3(1.4)$ \\
\hline Type III & $198(94.3)$ \\
\hline ECOG performance status & \\
\hline 0 & $168(80.0)$ \\
\hline 1 & $42(20.0)$ \\
\hline T stage * & \\
\hline T1 & $7(3.3)$ \\
\hline T2 & $29(13.8)$ \\
\hline T3 & $96(45.7)$ \\
\hline T4 & $78(37.2)$ \\
\hline N stage * & \\
\hline N0 & $21(10.0)$ \\
\hline N1 & $62(29.5)$ \\
\hline N2 & $108(51.4)$ \\
\hline N3 & $19(9.1)$ \\
\hline Clinical stage * & \\
\hline III & $117(55.7)$ \\
\hline IVa & $75(35.7)$ \\
\hline IVb & $18(8.6)$ \\
\hline Comorbidity & \\
\hline No & $154(73.3)$ \\
\hline Yes & $56(26.7)$ \\
\hline & \\
\hline
\end{tabular}

Abbreviations: WHO: World Health Organization. ECOG: Eastern Cooperative Oncology Group.

*The 7 th AJCC/UICC staging system.

91.4\%, $P=0.011$ ) (Figure 2B). The 5-year OS and PFS rates were $92.7 \%, 83.2 \%$, and $85.0 \%$ and $89.9 \%, 79.8 \%$, and $64.2 \%$ for patients with stage III, stage IVa, and stage IVb, respectively $(P=0.020)$ (Figure $2 \mathrm{C}),(P=0.012)$ (Figure 3A). The 5-year OS rate of patients treated with a CC regimen of cisplatin was higher than that of noncisplatin CC regimens (89.9\% vs. 69.4\%) $(P=0.036)$ (Figure 2D). The 5-year PFS rates were 90.2\%, 89.9\%, $89.2 \%$, and $78.2 \%$ for patients with stage N0, N1, N2, and N3 disease, respectively $(P=0.012)$ (Figure 3B). The 5 -year PFS rates for patients with $\mathrm{AC} v s$. without $\mathrm{AC}$ and CR patients vs. non-CR patients were $89.4 \%$ vs. $77.9 \%$ $(P=0.030)$ (Figure $3 \mathrm{C})$ and $88.5 \%$ vs. $69.8 \%(P=0.008)$ (Figure 3D), respectively.

Treatment failure occurred in 31 patients by the last follow-up. Local relapse occurred in 7 patients, regional relapse occurred in 8 patients, and locoregional relapse occurred in 1 patient. Distant failure was experienced by 15 patients. Patterns of treatment failure in NPC patients are listed in Table 3.

\section{Identification of prognostic factors}

We evaluated the following potential prognostic factors: patient age, patient gender, clinical stage, adjusted tumor (T) and lymph node (N) stage, NAC, CCRT, AC, comorbidities, dose of nimotuzumab, and tumor response at the end of treatment. Univariate analysis revealed that clinical stage and CCRT regimens were significant prognostic factors for OS, and N stage, clinical stage, and tumor response at the end of treatment were significant prognostic factors for PFS (Table 4). In multivariate analysis, stage IV was a poorer prognostic factor for OS, 
Table 2: Completion of treatment in 210 patients with LA NPC

\begin{tabular}{|l|l|}
\hline Treatment & $\mathbf{N}(\mathbf{\%})$ \\
\hline NAC regimens & \\
\hline TPF & $62(29.5)$ \\
\hline TP & $64(30.5)$ \\
\hline GP & $7(3.3)$ \\
\hline FP & $73(34.8)$ \\
\hline Other & $4(1.9)$ \\
\hline Cycle of NAC & \\
\hline 1 & $40(19.0)$ \\
\hline 2 & $80(38.1)$ \\
\hline $3-4$ & $90(42.9)$ \\
\hline CC regimens & \\
\hline Cisplatin & $198(94.3)$ \\
\hline Non-cisplatin & $12(5.7)$ \\
\hline Cycle of CC & \\
\hline 1 & $76(36.2)$ \\
\hline 2 & $120(57.1)$ \\
\hline$\geq 3$ & $14(6.7)$ \\
\hline Period of CC & \\
\hline Weekly & $12(5.7)$ \\
\hline 3 weeks & $198(94.3)$ \\
\hline AC & \\
\hline No & $115(54.8)$ \\
\hline Yes & $95(45.2)$ \\
\hline Fractional dose of h-R3 & \\
\hline 100 mg & $23(11.0)$ \\
\hline 200 mg & $187(89.0)$ \\
\hline Total dose of h-R3 & \\
\hline$<1200$ mg & $60(28.6)$ \\
\hline$\geq 1200$ mg & $150(71.4)$ \\
\hline Cycle of h-R3 & \\
\hline$<6$ weeks & $39(18.6)$ \\
\hline$\geq 6$ weeks & $171(81.4)$ \\
\hline & CC: \\
\hline
\end{tabular}

Abbreviations: NAC: neoadjuvant chemotherapy. CC: concurrent chemotherapy. AC: adjuvant chemotherapy. h-R3: nimotuzumab.

PFS and DMFS, as were CC regimen of non-cisplatin for OS and DMFS and non-CR for OS and RRFS (Table 5).

\section{Safety and toxicity}

The most common treatment-related acute adverse events included hematologic and non- hematologic toxicity (Table 6). During the period of NAC, hematologic toxicity was reported as grade 3 and worse in severity in 45 patients $(21.4 \%)$. Of these patients, 8 experienced neutropenic fever. The toxicities can be tolerated without delaying the chemotherapy and interrupting radiotherapy by GMSF treatment. The gastrointestinal toxicities were mild or moderate, and patients recovered rapidly with or without symptomatic medication. During the period of CCRT, the grade 3-4 hematologic toxicities and radiotherapy-related oral mucositis were reported in 14 patients $(6.7 \%)$ and 9 patients $(4.3 \%)$, respectively. Grade 3 dermatitis was observed in 5 patients within the RT field. No acneiform eruptions were found among these patients.

The most commonly observed late complication was xerostomia. However, the degree typically decreased over time. The degree of dry mouth in most patients was mild-to-moderate at the time of the last follow-up, and 123 patients did not complain of xerostomia. Finally, 79 patients developed either unilateral or bilateral hearing impairment, and 11 patients were found to have temporal lobe damage, which was diagnosed during follow-up based on magnetic resonance imaging. 
Table 3: Patterns of treatment failure in nasopharyngeal carcinoma patients

\begin{tabular}{|l|l|}
\hline Sites & Number of patients $(\boldsymbol{n}=\mathbf{3 1})$ \\
\hline Local relapse only & 7 \\
\hline Regional relapse only & 8 \\
\hline Local and regional failure & 1 \\
\hline Regional and distant failure & 2 \\
\hline Distant failure only & 13 \\
\hline Lung metastasis only & 3 \\
\hline Bone metastasis only & 2 \\
\hline Liver metastasis only & 2 \\
\hline Lung, liver, bone and other & 6 \\
\hline
\end{tabular}

\section{DISCUSSION}

With further research on the molecular mechanism of tumorigenesis and tumor development, targeted molecular therapy in patients with NPC will become the research focus. Overexpression of EGFR was detected in $94 \%$ of patients with NPC [18]. Cetuximab is a common anti-EGFR monoclonal antibody drug. It has a good curative effect in the treatment of NPC, with a 2-year
PFS of $86.5 \%$ to $89.3 \%$ and a 3 -year OS of $90.9 \%$ [22], but severe oral mucositis and itchy acneiform rash limit its application in NPC. To minimize cetuximab-related toxicities, a novel EGFR-targeted agent without toxicities is warranted.

Nimotuzumab, a humanized immunoglobulin G1 (IgG1) isotype monoclonal antibody with a unique safety profile and low skin toxicity, has been approved for the treatment of non-NPC HNSCC $[16,30]$. The advantage
A

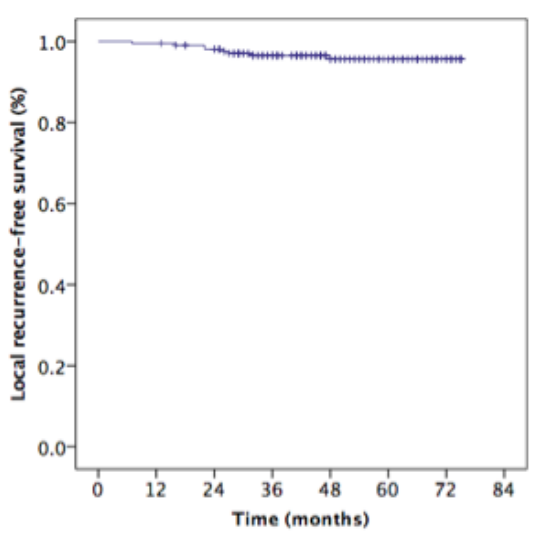

B

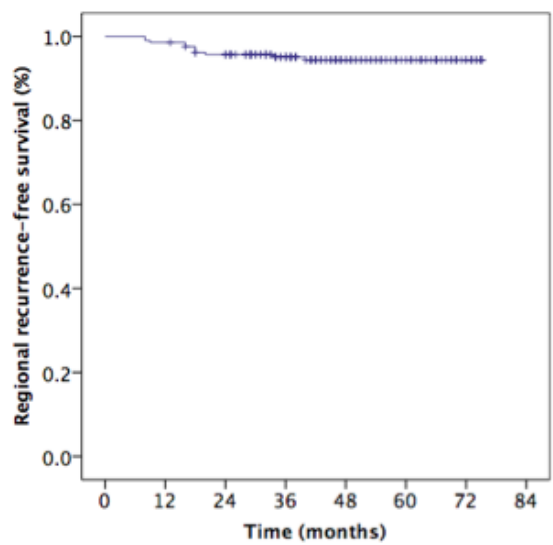

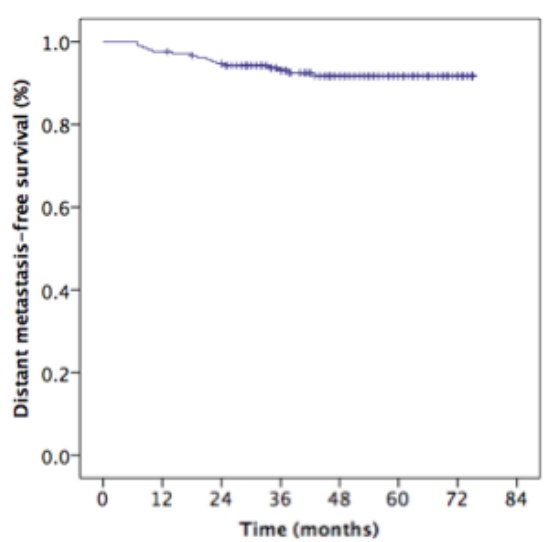

D

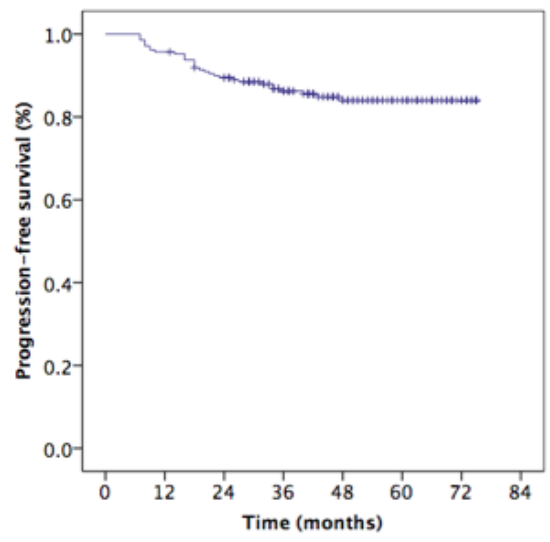

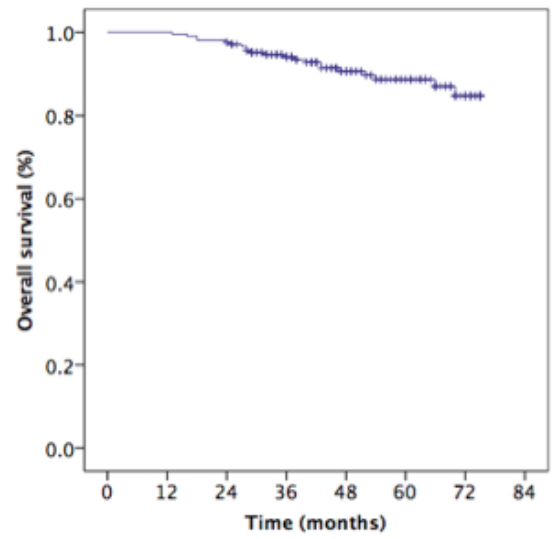

Figure 1: Kaplan-Meier estimates of the survival in patients with nasopharyngeal carcinoma. A. Local recurrence-free survival, B. regional recurrence-free survival, C. distance metastasis-free survival, D. progression-free survival, and E. overall survival. 
Table 4: Univariate analysis of prognostic factors on OS and PFS in LA NPC patients

\begin{tabular}{|c|c|c|c|c|c|}
\hline Characteristic & $N$ & 5-year OS (\%) & $P$ & 5-year PFS (\%) & $P$ \\
\hline Gender & & & 0.319 & & 0.058 \\
\hline Male & 154 & 86.6 & & 80.7 & \\
\hline Female & 17 & 94.0 & & 92.6 & \\
\hline Age (years) & & & 0.124 & & 0.870 \\
\hline$<50$ & 131 & 90.5 & & 83.6 & \\
\hline$\geq 50$ & 79 & 85.4 & & 84.8 & \\
\hline T stage * & & & 0.080 & & 0.361 \\
\hline $\mathrm{T} 1$ & 7 & 100.0 & & 85.7 & \\
\hline $\mathrm{T} 2$ & 29 & 90.1 & & 81.4 & \\
\hline $\mathrm{T} 3$ & 96 & 91.0 & & 88.4 & \\
\hline $\mathrm{T} 4$ & 78 & 83.8 & & 79.3 & \\
\hline N stage $*$ & & & 0.884 & & 0.012 \\
\hline N0 & 21 & 90.2 & & 85.7 & \\
\hline N1 & 62 & 89.9 & & 91.6 & \\
\hline $\mathrm{N} 2$ & 108 & 89.2 & & 83.6 & \\
\hline N3 & 19 & 78.2 & & 60.8 & \\
\hline Clinical stage * & & & 0.012 & & 0.012 \\
\hline III & 117 & 92.7 & & 89.9 & \\
\hline IVA & 75 & 83.2 & & 79.8 & \\
\hline IVB & 18 & 85.0 & & 64.2 & \\
\hline Comorbidity & & & 0.193 & & 0.640 \\
\hline No & 154 & 89.5 & & 83.2 & \\
\hline Yes & 56 & 86.3 & & 86.4 & \\
\hline NAC regimens & & & 0.257 & & 0.462 \\
\hline TPF & 62 & 89.9 & & 79.3 & \\
\hline $\mathrm{TP}$ & 64 & 90.9 & & 89.8 & \\
\hline GP\# & 7 & - & & - & \\
\hline FP & 73 & 83.3 & & 82.1 & \\
\hline Other & 4 & 100.0 & & & \\
\hline Cycle of NAC & & & 0.447 & & 0.169 \\
\hline 1 & 40 & 84.8 & & 74.9 & \\
\hline 2 & 80 & 89.3 & & 85.2 & \\
\hline $3-4$ & 90 & 89.8 & & 87.0 & \\
\hline Period of CC & & & 0.992 & & 0.781 \\
\hline Weekly & 12 & 85.7 & & 84.1 & \\
\hline 3 weeks & 198 & 88.9 & & 83.3 & \\
\hline $\mathrm{CC}$ regimens & & & 0.036 & & 0.230 \\
\hline Cisplatin & 198 & 89.9 & & 84.6 & \\
\hline Non-cisplatin & 12 & 69.4 & & 75.0 & \\
\hline $\mathrm{AC}$ & & & 0.369 & & 0.030 \\
\hline No & 115 & 91.6 & & 89.4 & \\
\hline Yes & 95 & 86.3 & & 77.9 & \\
\hline Fractional dose of h-R3 & & & 0.966 & & 0.896 \\
\hline $100 \mathrm{mg}$ & 23 & 86.7 & & 87.0 & \\
\hline $200 \mathrm{mg}$ & 186 & 88.7 & & 83.4 & \\
\hline Total dose of h-R3 & & & 0.976 & & 0.459 \\
\hline$<1200 \mathrm{mg}$ & 60 & 83.6 & & 85.2 & \\
\hline$\geq 1200 \mathrm{mg}$ & 120 & 84.3 & & 90.0 & \\
\hline Tumor response & & & 0.270 & & 0.008 \\
\hline $\mathrm{CR}$ & 163 & 90.8 & & 88.5 & \\
\hline Non-CR & 47 & 81.4 & & 69.8 & \\
\hline
\end{tabular}

Abbreviations: NAC: neoadjuvant chemotherapy. CC: concurrent chemotherapy. AC: adjuvant chemotherapy. h-R3: nimotuzumab. CR: complete response. *The 7th AJCC/UICC staging system. \# Patients, who treated with GP, lived fewer than 5 years 
Table 5: Multivariate analysis of prognostic factors in LA NPC patients

\begin{tabular}{|l|l|c|c|c|}
\hline \multicolumn{1}{|c|}{ Characteristic } & HR & 95\% CI & P-value \\
\hline OS & CC regimen of cisplatin $v s$. non-cisplatin & 0.256 & $0.074-0.886$ & 0.031 \\
\hline & III $v s$. IV $*$ & 0.291 & $0.113-0.754$ & 0.011 \\
\hline PFS & III $v s$. IV $*$ & 0.438 & $0.213-0.899$ & 0.024 \\
\hline & CR $v s$. non-CR & 0.441 & $0.210-0.926$ & 0.031 \\
\hline LRFS & - & - & - & - \\
\hline RRFS & CR $v s$. non-CR & 0.300 & $0.091-0.994$ & 0.049 \\
\hline & With $v s$. without AC & 0.222 & $0.047-1.043$ & 0.057 \\
\hline DMFS & CC regimen of cisplatin $v s$. non-cisplatin & 0.207 & $0.058-0.734$ & 0.015 \\
\hline & III $v s$. IV $*$ & 0.166 & $0.047-0.583$ & 0.005 \\
\hline
\end{tabular}

Abbreviations: OS: overall survival. PFS: progression-free survival. LRFS: local recurrence-free survival. RRFS: regional recurrence-free survival. DMFS: distant metastasis-free survival. CC: concurrent chemotherapy. AC: adjuvant chemotherapy. *The 7th AJCC/UICC staging system.

A

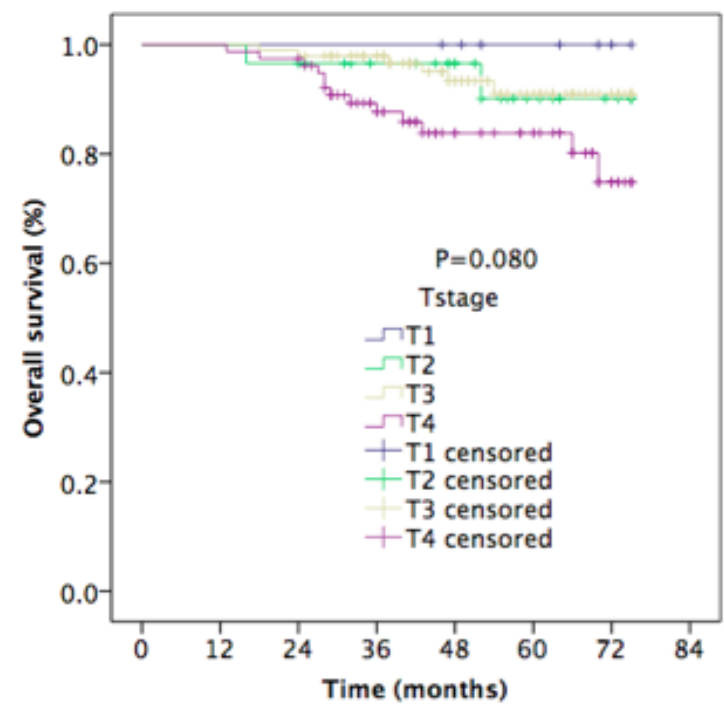

C

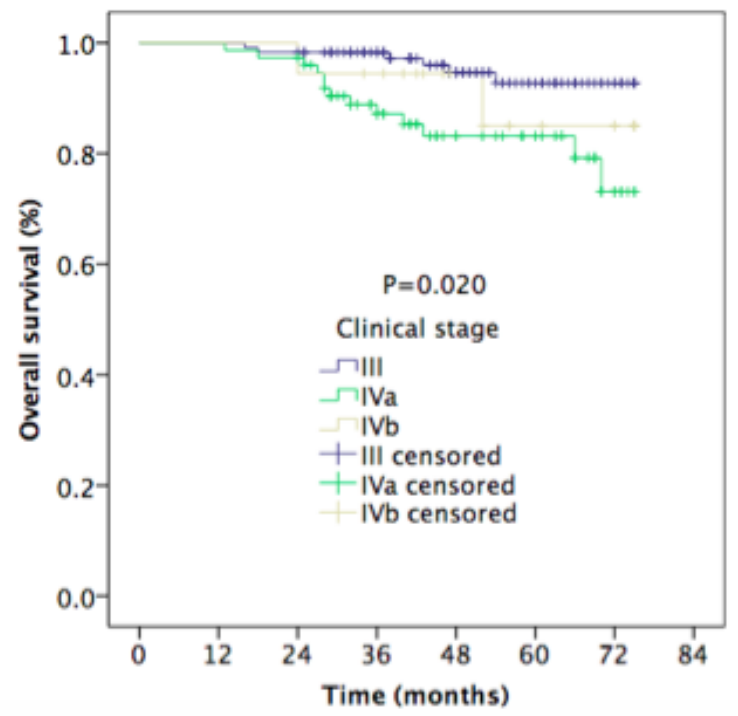

B

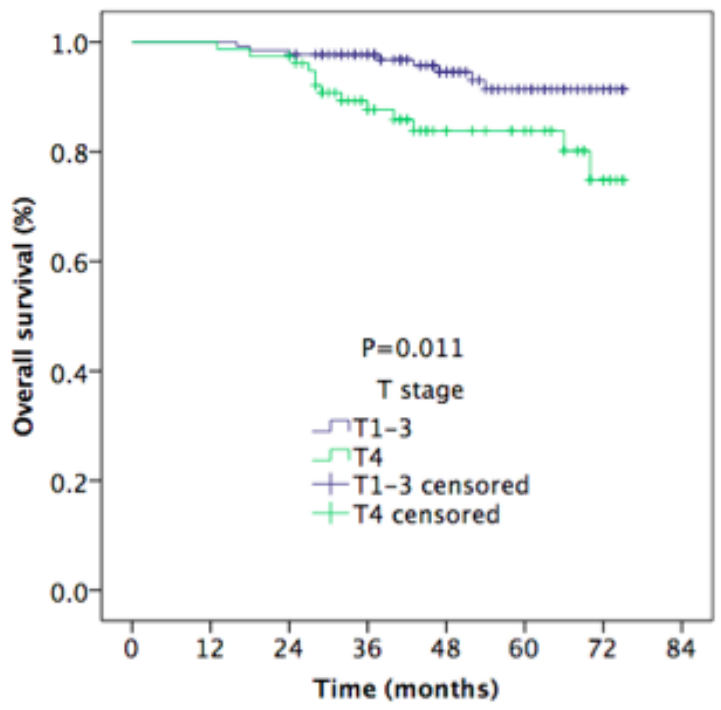

D

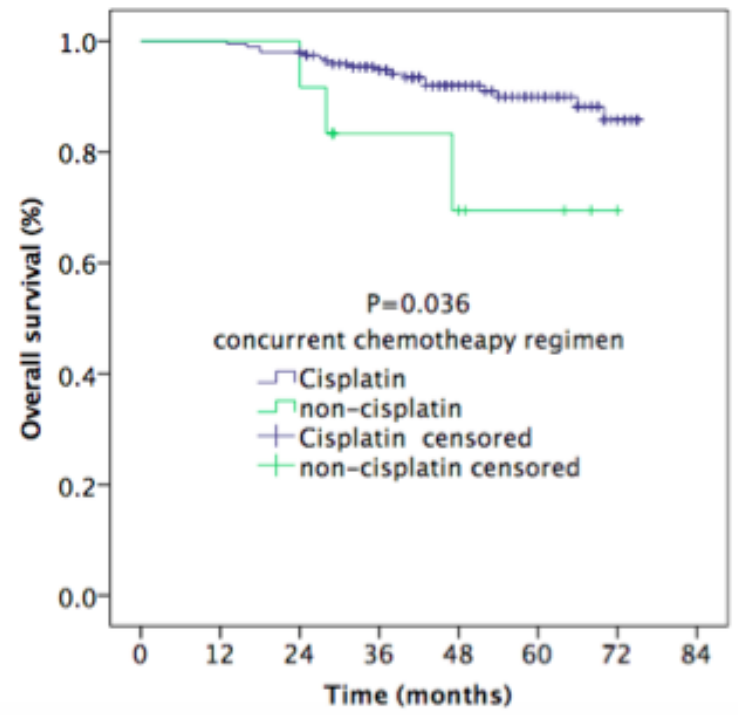

Figure 2: Kaplan-Meier estimates of the overall survival in nasopharyngeal carcinoma patients for different variable. A. Overall survival for T stages, B. overall survival for stage T4 vs. T1-T3, C. overall survival for clinical stage, and D. overall survival for concurrent chemotherapy regimens. 
Table 6: Toxicity of nimotuzumab plus NAC followed by CCRT with or without AC

\begin{tabular}{|l|c|c|c|c|c|c|c|c|c|c|}
\hline \multirow{2}{*}{\multicolumn{1}{c|}{ Adverse events }} & \multicolumn{4}{c|}{ During the period of NAC } & \multicolumn{4}{c|}{ During the period of CCRT } \\
\cline { 2 - 12 } & $\mathbf{0}$ & $\mathbf{1}$ & $\mathbf{2}$ & $\mathbf{3}$ & $\mathbf{4}$ & $\mathbf{0}$ & $\mathbf{1}$ & $\mathbf{2}$ & $\mathbf{3}$ & $\mathbf{4}$ \\
\hline White blood cell & 63 & 57 & 45 & 35 & 10 & 131 & 36 & 29 & 9 & 5 \\
\hline Leukocytopenia & 72 & 52 & 41 & 32 & 13 & 132 & 34 & 31 & 10 & 3 \\
\hline Anemia & 180 & 22 & 6 & 2 & 0 & 109 & 55 & 39 & 7 & 0 \\
\hline Thrombocytopenia & 184 & 17 & 6 & 3 & 0 & 147 & 38 & 23 & 2 & 0 \\
\hline Liver function & 198 & 8 & 4 & 0 & 0 & 192 & 15 & 3 & 0 & 0 \\
\hline Renal function & 203 & 7 & 0 & 0 & 0 & 206 & 3 & 1 & 0 & 0 \\
\hline Mucositis & 175 & 27 & 8 & 0 & 0 & 44 & 84 & 73 & 7 & 2 \\
\hline Dermatitis & 210 & 0 & 0 & 0 & 0 & 0 & 138 & 67 & 5 & 0 \\
\hline Diarrhea & 197 & 9 & 3 & 1 & 0 & 202 & 6 & 2 & 0 & 0 \\
\hline Nausea/vomiting & 157 & 29 & 17 & 5 & 2 & 172 & 25 & 12 & 1 & 0 \\
\hline
\end{tabular}

Abbreviations: NAC: neoadjuvant chemotherapy. CCRT: concurrent chemoradiotherapy.

A

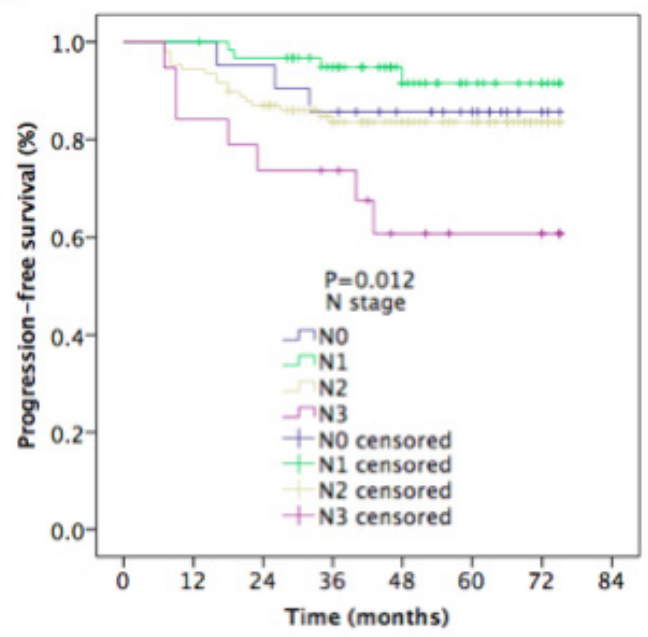

C

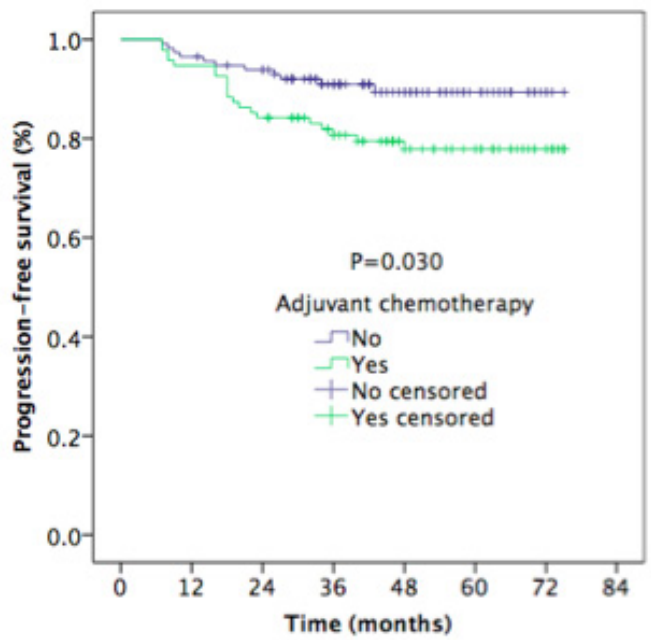

B

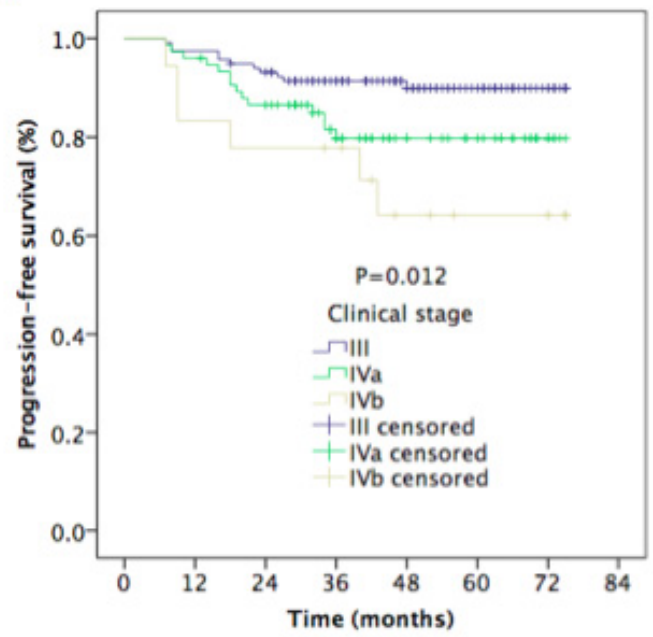

D

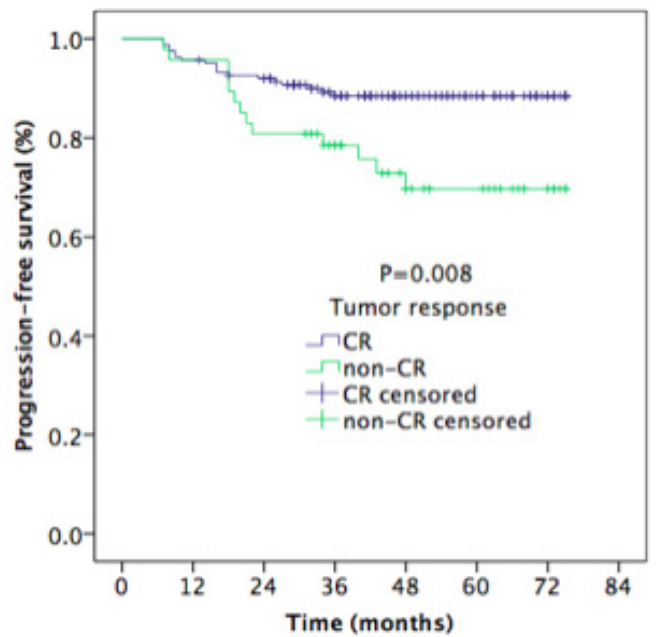

Figure 3: Kaplan-Meier estimates of the progression-free survival in nasopharyngeal carcinoma patients for univariate. A. Progression-free survival for N stages, B. progression-free survival for clinical stages, C. progression-free survival of patients with or without AC, and D. progression-free survival for tumor response. 
of the drug is that the affinity constant is lower than that of cetuximab, allowing for high tumor uptake and low normal-tissue uptake [31]. Nimotuzumab requires bivalent binding for stable attachment, which makes the agent selectively bind to tumors with moderate-to-high EGFR levels. When EGFR expression is low, as in tissue, cetuximab still has high binding ability because of its higher affinity constant [31]. Our experiment confirmed that nimotuzumab sensitizes nasopharyngeal carcinoma cell line CNE-2 in vitro to RT and can reduce cancer cell proliferation, induce cell apoptosis, and change cell cycle distribution [32]. All these effects indicate that nimotuzumab plus RT can be utilized in the design of the clinical trial of NPC.

To date, only four small-scale studies on adding nimotuzumab to RT or CCRT for NPC patients have been conducted. In a retrospective paired study by Li et al [33], the OS and PFS rates for the nimotuzumab/RT treatment group were lower than those for cisplatin/RT treatment group, but in the stage II or the older than 60 years subgroups, no significant differences were seen for OS and PFS. Zhai et al reported that the addition of nimotuzumab to IMRT showed promising locoregional control and survival outcomes for LA NPC patients [29]. Huang et al [34] and Liu et al [28] found that concurrent administration of nimotuzumab and CCRT yielded encouraging survival outcomes in LA NPC patients, with tolerable treatment-related toxicity. For the first two studies, because of the severe acute sequela of CCRT, nimotuzumab, as a preferred substrate for cisplatin, increased the quality of life in selected NPC patients, with similar treatment outcomes. However, in the last two studies, nimotuzumab added into the intensive modality of NAC followed by CCRT improved the survival of LA NPC patients but with normal-tissue damage. Those outcomes will be the direction of further research.

This study investigated the efficacy and safety of adding nimotuzumab to NAC followed by CCRT for

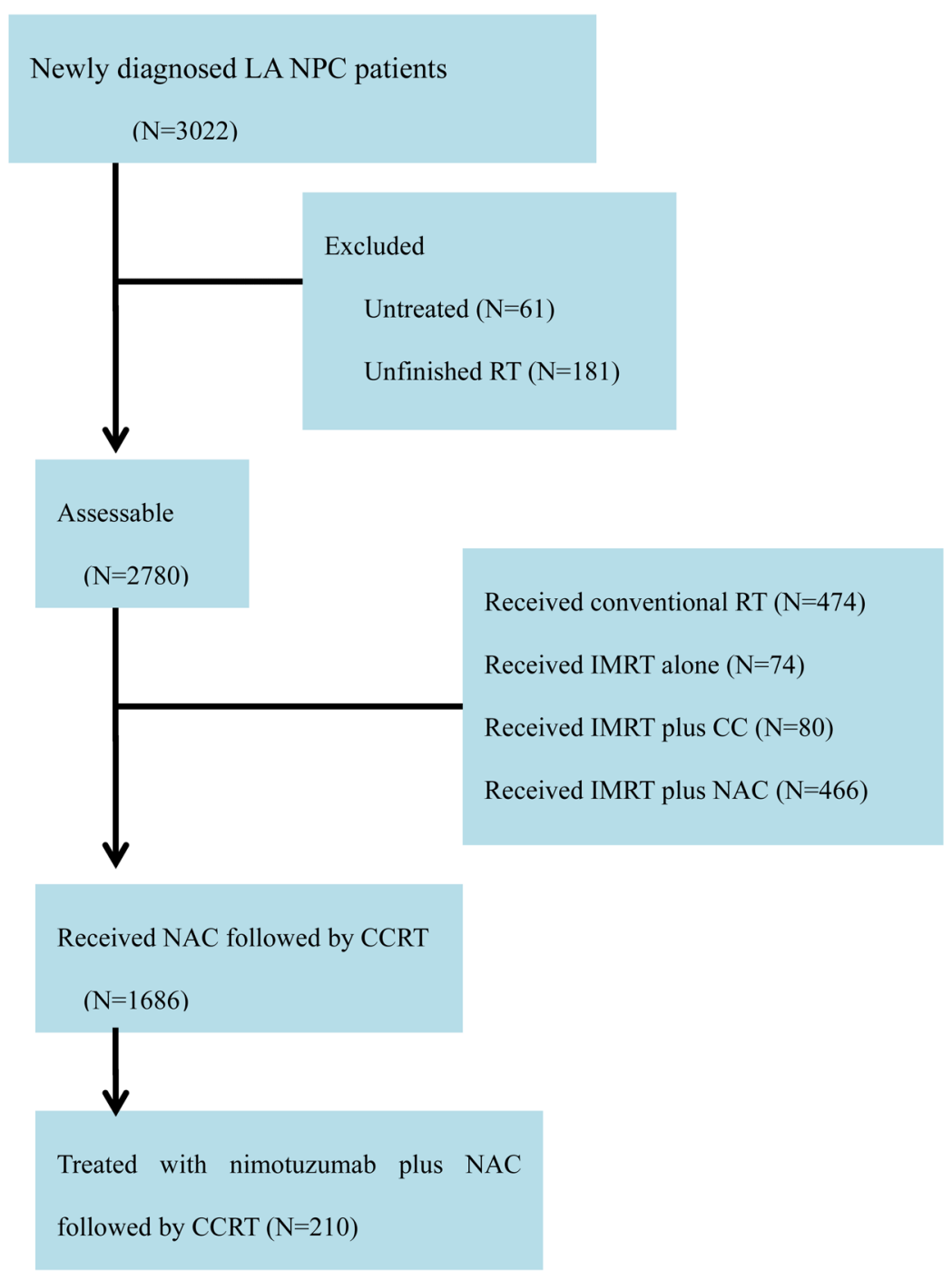

Figure 4: Flowchart of patients. Abbreviations: NPC: Nasopharyngeal carcinoma; RT: Radiotherapy. 
LA NPC patients. The study showed promising clinical outcomes, with a 5-year LRFS of 95.6\%, a 5-year RRFS of $94.4 \%$, a 5-year DMFS of 91.7\%, a 5-year PFS of $84.0 \%$, and a 5 -year OS of $88.7 \%$. Univariate analysis revealed that clinical stage and CCRT regimens are significant prognostic factors for OS, and $\mathrm{N}$ stage, clinical stage, and tumor response at the end of treatment are significant prognostic factor for PFS. Multivariate analysis indicated that stage IV is a poorer prognostic factor for OS, PFS and DMFS, as stage N2 to N3 is for PFS and DMFS, a CCRT regimen of non-cisplatin is for OS and DMFS, and a non-CR is for OS and RRFS. Although $34.7 \%$ of patients experienced grade $\geq 3$ hematologic toxicity and $12.0 \%$ experienced grade $\geq 3$ radiotherapy-related oral mucositis, only 5 patients were observed with grade 3 dermatitis within the RT field. No acneiform eruptions were found among these patients.

We found that nimotuzumab plus NAC before CCRT with or without AC in the treatment of LA NPC patients is safe and effective. However, because of the retrospective nature of the study, our results should be regarded as preliminary.

\section{CONCLUSION}

We observed that the administration of nimotuzumab with NAC followed by CCRT in LA NPC patients was tolerated and showed promising clinic outcomes. Further randomized, controlled, multicenter phase III clinical trials are needed to confirm the therapeutic gain.

\section{PATIENTS AND METHODS}

\section{Pretreatment}

The patients enrolled into this study were hospitalized from May 2008 to April 2014 in the Department of Radiation Oncology, Zhejiang Cancer Hospital. The retrospective study was approved by the medical ethics committee of Zhejiang Cancer Hospital. The eligible patients met the following criteria: (i) histologically proven LA NPC, (ii) Eastern Cooperative Oncology Group performance status $\leq 1$, (iii) completion of radical IMRT, (iv) received nimotuzumab plus NAC before CCRT, and (v) no previous anti-cancer treatment.

Patients had pretreatment evaluations that included complete histories, physical examinations, hematology and biochemistry profiles, chest radiographs, sonography of the abdomen, bone scans, magnetic response images of the nasopharynx, and nasopharyngoscopies. All patients were staged per the 2010 American Joint Committee on Cancer staging system. Tumor histology was classified per the World Health Organization classification.

The flowchart of patients is shown in Figure 4. A total of 3022 newly diagnosed LA NPC patients were registered at Zhejiang Cancer Hospital. A total of 210 NPC patients treated with nimotuzumab plus NAC followed by CCRT were enrolled into this study. All patients received definitive IMRT with or without AC.

\section{Radiotherapy}

All patients were immobilized in the supine position with thermoplastic masks. Computed tomography scans with intravenous contrast $(2.5 \mathrm{~mm}$ slices from the head to $2 \mathrm{~cm}$ below the sternoclavicular joints) were performed for planning. All patients underwent radical IMRT with a simultaneous integrated boost technique that used 6-MV photons. The prescribed radiation doses were $69 \mathrm{~Gy}$ or 72 Gy to planning gross target volume (PGTV)nx, 66 Gy to 69 Gy to PGTVnd, 63 Gy to 66 Gy to planning target volume (PTV)nx, 60 Gy to 63 Gy to PTV1, and 51 Gy to 54 Gy to PTV2, delivered in 30 or 33 fractions. Radiation was delivered once daily, in five fractions per week, over 6 to 6.5 weeks for IMRT planning. The dose to organs at risk was limited based on the Radiation Therapy Oncology Group 0225 protocol.

\section{Target treatment}

Nimotuzumab was administered concomitantly with induction chemotherapy and /or RT at a dose of $100 \mathrm{mg}$ or $200 \mathrm{mg}$ weekly, which was diluted in $250 \mathrm{~mL}$ of saline to obtain a $100-\mathrm{mg}$ or $200-\mathrm{mg}$ suspension and intravenously infused over 1 hour. All patients received 3 to 17 weeks of nimotuzumab during the treatment.

\section{Chemotherapy}

All patients were given one to four cycles of platinum-based induction chemotherapy. The most common induction regimens included TPF (docetaxel 60 $\mathrm{mg} / \mathrm{m}^{2} /$ day on day 1 , cisplatin $25 \mathrm{mg} / \mathrm{m}^{2} /$ day on days 1 to 3 , and 5 -fluorouracil $500 \mathrm{mg} / \mathrm{m}^{2} /$ day on days 1 to 3 ), $\mathrm{TP}$ (docetaxel $60 \mathrm{mg} / \mathrm{m}^{2} /$ day on day 1, cisplatin $25 \mathrm{mg} / \mathrm{m}^{2} /$ day on days 1 to 3 ), GP (gemcitabine $1,000 \mathrm{mg} / \mathrm{m}^{2} /$ day on days 1 and 8 , cisplatin $25 \mathrm{mg} / \mathrm{m}^{2} /$ day on days 1 to 3 ), and FP (cisplatin $25 \mathrm{mg} / \mathrm{m}^{2} /$ day on days 1 to 3 , and 5 -fluorouracil $500 \mathrm{mg} / \mathrm{m}^{2} /$ day on days 1 to 3 ).

NPC patients underwent $\geq 1$ cycle concurrent chemotherapy with cisplatin $\left(80 \mathrm{mg} / \mathrm{m}^{2}\right)$ for 3 days, and 95 patients received two to three courses of adjuvant chemotherapy with FP regimen 3 weeks after RT.

\section{Patient evaluation and follow-up}

The assessment of tumor response was performed thrice after the completion of induction chemotherapy, at 
the end of IMRT, and 3 months after irradiation, which was based on MRI and nasopharynx fiberscope per the Response Evaluation Criteria for Solid Tumors. Systemic chemotherapy adverse effects were graded per the National Cancer Institute Common Toxicity Criteria (NCI CTCAE, Version 3.0), and RT-induced toxicities were scored per the Acute and Late Radiation Morbidity Scoring Criteria of the Radiation Therapy Oncology Group.

All the subjects underwent weekly examinations for treatment response and toxicities during RT. Patients were followed every 3 months in the first 2 years, every 6 months from the third to the fifth year, and then annually. Each follow-up included careful examination of the nasopharynx and neck nodes by an experienced doctor. MRI scan of the nasopharynx, nasopharynx fiberscope, chest computed tomography radiograph, and ultrasound of abdomen were performed 3 months after the completion of RT and every 6 to 12 months thereafter. Additional examinations were performed when indicated to evaluate local relapse or distant metastasis.

\section{Statistical analysis}

Survival curves were generated by use of the Kaplan-Meier method. The curves were compared by use of log-rank tests. Multivariate analysis was performed by use of Cox regression models to identify significant prognostic factors. Hazard ratios (HRs) and 95\% confidence intervals (CIs) were calculated for each prognostic factor. IBM SPSS Statistics Version 19.0 was used for all data analysis. A $P<0.05$ was considered statistically significant. Survival time was calculated from the date of diagnosis to the most recent follow-up or to either the date of relapse (event-free, local recurrencefree, or distant metastasis-free) or death (overall survival). After recurrence or metastasis, patients were given salvage therapy as determined by their physicians.

\section{FUNDING}

This study was supported by grants from the Medical Science Foundation of Zhejiang Health Bureau (No. 2013KYB033, No. 2009B026, No. 2006A016, No. 2005B012, No. 2004B014), National Natural Science Foundation of China (No. 81502646, No. 81502647).

\section{CONFLICTS OF INTEREST}

The authors declare that there are no conflicts of interest.

\section{REFERENCES}

1. Tang LL, Chen WQ, Xue WQ, He YQ, Zheng RS, Zeng YX, Jia WH. Global trends in incidence and mortality of nasopharyngeal carcinoma. Cancer Lett. 2016; 374:22-30.

2. Chen L, Mao YP, Xie FY, Liu LZ, Sun Y, Tian L, Tang LL, Lin AH, Li L, Ma J. The seventh edition of UICC/ AJCC staging system for nasopharyngeal carcinoma is prognostically useful for patients treated with intensitymodulated radiotherapy from an endemic area in China. Radiother Oncol. 2012;104:331-337.

3. Wee J. Nasopharyngeal cancer: a promising future. Lancet Oncol. 2012; 13:116-18.

4. Lai SZ, Li WF, Chen L, Luo W, Chen YY, Liu LZ, Sun Y, Lin AH, Liu MZ, Ma J. How does intensitymodulated radiotherapy versus conventional twodimensional radiotherapy influence the treatment results in nasopharyngeal carcinoma patients? Int J Radiat Oncol Biol Phys. 2011; 80:661-68.

5. Al-Sarraf M, Reddy MS. Nasopharyngeal carcinoma. Curr Treat Options Oncol. 2002; 3:21-32.

6. Al-Sarraf M, LeBlanc M, Giri PG, Fu KK, Cooper J, Vuong T, Forastiere AA, Adams G, Sakr WA, Schuller DE, Ensley JF. Chemoradiotherapy versus radiotherapy in patients with advanced nasopharyngeal cancer: phase III randomized Intergroup study 0099. J Clin Oncol. 1998; 16:1310-17.

7. Lee AW, Tung SY, Chua DT, Ngan RK, Chappell R, Tung R, Siu L, Ng WT, Sze WK, Au GK, Law SC, O’Sullivan $\mathrm{B}$, Yau TK, et al. Randomized trial of radiotherapy plus concurrent-adjuvant chemotherapy vs radiotherapy alone for regionally advanced nasopharyngeal carcinoma. J Natl Cancer Inst. 2010; 102:1188-98.

8. Baujat B, Audry H, Bourhis J, Chan AT, Onat H, Chua DT, Kwong DL, Al-Sarraf M, Chi KH, Hareyama M, Leung SF, Thephamongkhol K, Pignon JP, and MAC-NPC Collaborative Group. Chemotherapy in locally advanced nasopharyngeal carcinoma: an individual patient data metaanalysis of eight randomized trials and 1753 patients. Int $\mathrm{J}$ Radiat Oncol Biol Phys. 2006; 64:47-56.

9. OuYang PY. Xie C, Mao YP, Zhang Y, Liang XX, Su Z, Liu Q, Xie FY. Significant efficacies of neoadjuvant chemotherapy and adjuvant chemotherapy for nasopharyngeal carcinoma by meta-analysis of published literature-based randomized, control trials. Ann Oncol. 2013; 24:2136-46.

10. Chen YP, Guo R, Liu N, Liu X, Mao YP, Tang LL, Zhou GQ, Lin AH, Sun Y, Ma J. Efficacy of the additional neoadjuvant chemotherapy to concurrent chemoradiotherapy for patients with locoregionally advanced nasopharyngeal carcinoma: a Bayesian network meta-analysis of randomized controlled trials. J Cancer. 2015; 6:883-92.

11. Wang M, Tian H, Li G, Ge T, Liu Y, Cui J, Han F. Significant benefits of adding neoadjuvant chemotherapy before concurrent chemoradiotherapy for locoregionally advanced nasopharyngeal carcinoma: a meta-analysis of randomized controlled trials. Oncotarget. 2016; 7:4837548390. https://doi.org/10.18632/oncotarget.10237 
12. Hui EP, Ma BB, Leung SF, King AD, Mo F, Kam MK, Yu BK, Chiu SK, Kwan WH, Ho R, Chan I, Ahuja AT, Zee BC, Chan AT. Randomized phase II trial of concurrent cisplatinradiotherapy with or without neoadjuvant docetaxel and cisplatin in advanced nasopharyngeal carcinoma. J Clin Oncol. 2009; 27:242-49.

13. Fountzilas G, Ciuleanu E, Bobos M, Kalogera-Fountzila A, Eleftheraki AG, Karayannopoulou G, Zaramboukas T, Nikolaou A, Markou K, Resiga L, Dionysopoulos D, Samantas E, Athanassiou H, et al. Induction chemotherapy followed by concomitant radiotherapy and weekly cisplatin versus the same concomitant chemoradiotherapy in patients with nasopharyngeal carcinoma: a randomized phase II study conducted by the Hellenic Cooperative Oncology Group (HeCOG) with biomarker evaluation. Ann Oncol. 2012; 23:427-35.

14. Huang PY, Cao KJ, Guo X, Mo HY, Guo L, Xiang YQ, Deng MQ, Qiu F, Cao SM, Guo Y, Zhang L, Li NW, Sun $\mathrm{R}$, et al. A randomized trial of induction chemotherapy plus concurrent chemoradiotherapy versus induction chemotherapy plus radiotherapy for locoregionally advanced nasopharyngeal carcinoma. Oral Oncol. 2012; 48:1038-44.

15. Wu F, Wang R, Lu H, Wei B, Feng G, Li G, Liu M, Yan $\mathrm{H}$, Zhu J, Zhang Y, Hu K. Concurrent chemoradiotherapy in locoregionally advanced nasopharyngeal carcinoma: treatment outcomes of a prospective, multicentric clinical study. Radiother Oncol. 2014; 112:106-11.

16. Reddy BK, Lokesh V, Vidyasagar MS, Shenoy K, Babu KG, Shenoy A, Naveen T, Joseph B, Bonanthaya R, Nanjundappa, Bapsy PP, Loknatha, Shetty J, et al. Nimotuzumab provides survival benefit to patients with inoperable advanced squamous cell carcinoma of the head and neck: a randomized, open-label, phase IIb, 5-year study in Indian patients. Oral Oncol. 2014; 50:498-505.

17. Herbst RS, Shin DM. Monoclonal antibodies to target epidermal growth factor receptor-positive tumors: a new paradigm for cancer therapy. Cancer. 2002; 94:1593-611.

18. Chua DT, Nicholls JM, Sham JS, Au GK. Prognostic value of epidermal growth factor receptor expression in patients with advanced stage nasopharyngeal carcinoma treated with induction chemotherapy and radiotherapy. Int J Radiat Oncol Biol Phys. 2004; 59:11-20.

19. Köhler J, Schuler M. Afatinib, erlotinib and gefitinib in the first-line therapy of EGFR mutation-positive lung adenocarcinoma: a review. Onkologie. 2013; 36:510-18.

20. Yewale C, Baradia D, Vhora I, Patil S, Misra A. Epidermal growth factor receptor targeting in cancer: a review of trends and strategies. Biomaterials. 2013; 34:8690-707.

21. Bonner JA, Harari PM, Giralt J, Cohen RB, Jones CU, Sur RK, Raben D, Baselga J, Spencer SA, Zhu J, Youssoufian H, Rowinsky EK, Ang KK. Radiotherapy plus cetuximab for locoregionally advanced head and neck cancer: 5-year survival data from a phase 3 randomised trial, and relation between cetuximab-induced rash and survival. Lancet
Oncol. 2010; 11:21-28.

22. Feng HX, Guo SP, Li GR, Zhong WH, Chen L, Huang LR, Qin HY, Baselga J, Spencer SA, Zhu J, Youssoufian H, Rowinsky EK, Ang KK. Toxicity of concurrent chemoradiotherapy with cetuximab for locoregionally advanced nasopharyngeal carcinoma. Med Oncol. 2014; 31:170-78.

23. He X, Xu J, Guo W, Jiang X, Wang X, Zong D. Cetuximab in combination with chemoradiation after induction chemotherapy of locoregionally advanced nasopharyngeal carcinoma: preliminary results. Future Oncol. 2013; 9:1459-67.

24. Talavera A, Friemann R, Gómez-Puerta S, MartinezFleites C, Garrido G, Rabasa A, López-Requena A, Pupo A, Johansen RF, Sánchez O, Krengel U, Moreno E. Nimotuzumab, an antitumor antibody that targets the epidermal growth factor receptor, blocks ligand binding while permitting the active receptor conformation. Cancer Res. 2009; 69:5851-59.

25. Crombet T, Osorio M, Cruz T, Roca C, del Castillo R, Mon R, Iznaga-Escobar N, Figueredo R, Koropatnick J, Renginfo E, Fernández E, Alvárez D, Torres $\mathrm{O}$, et al. Use of the humanized anti-epidermal growth factor receptor monoclonal antibody h-R3 in combination with radiotherapy in the treatment of locally advanced head and neck cancer patients. J Clin Oncol. 2004; 22:1646-54.

26. Crombet $\mathrm{T}$, Torres L, Neninger E, Catalá M, Solano ME, Perera A, Torres O, Iznaga N, Torres F, Pérez R, Lage A, Alvárez $\mathrm{D}$, Torres $\mathrm{O}$, et al. Pharmacological evaluation of humanized anti-epidermal growth factor receptor, monoclonal antibody h-R3, in patients with advanced epithelial-derived cancer. J Immunother. 2003; 26:139-48.

27. Du F, Zheng Z, Shi S, Jiang Z, Qu T, Yuan X, Sun Y, Song Y, Yang L, Zhao J, Wang J, Chi Y. S-1 and cisplatin with or without nimotuzumab for patients with untreated unresectable or metastatic gastric cancer: A randomized, open-label phase 2 trial. Medicine (Baltimore). 2015; 94:e958.

28. Liu ZG, Zhao Y, Tang J, Zhou YJ, Yang WJ, Qiu YF, Wang H. Nimotuzumab combined with concurrent chemoradiotherapy in locally advanced nasopharyngeal carcinoma: a retrospective analysis. Oncotarget. 2016; 7:24429-24435. https://doi.org/10.18632/oncotarget.8225.

29. Zhai RP, Ying HM, Kong FF, Du CR, Huang S, Zhou JJ, $\mathrm{Hu} \mathrm{CS}$. Experience with combination of nimotuzumab and intensity-modulated radiotherapy in patients with locoregionally advanced nasopharyngeal carcinoma. Onco Targets Ther. 2015; 8:3383-90.

30. Rodríguez MO, Rivero TC, del Castillo Bahi R, Muchuli CR, Bilbao MA, Vinageras EN, Alert J, Galainena JJ, Rodríguez E, Gracias E, Mulén B, Wilkinson B, de Armas EL, et al. Nimotuzumab plus radiotherapy for unresectable squamous-cell carcinoma of the head and neck. Cancer Biol Ther. 2010; 9:343-49.

31. Ramakrishnan MS, Eswaraiah A, Crombet T, Piedra P, 
Saurez G, Iyer H, Arvind AS, Arvind AS. Nimotuzumab, a promising therapeutic monoclonal for treatment of tumors of epithelial origin. MAbs. 2009; 1:41-48.

32. Hua YH, Ma SL. FU ZF, Hu QY, Du LB, Jiang H. Effect of nimotuzumab on the radiation sensitivity of nasopharyngeal carcinoma cell line CNE-2. Chin J Zhejiang Med. 2011; 33:836-39.

33. Li HM, Li P, Qian YJ, Wu X, Xie L, Wang F, Zhang H, Liu L. A retrospective paired study: efficacy and toxicity of nimotuzumab versus cisplatin concurrent with radiotherapy in nasopharyngeal carcinoma. BMC Cancer. 2016; 16:94655.
34. Huang JF, Zhang FZ, Zou QZ, Zhou LY, Yang B, Chu JJ, Yu JH, Zhang HW, Yuan XP, Tai GM, Liu FJ, Ma CC. Induction chemotherapy followed by concurrent chemoradiation and nimotuzumab for locoregionally advanced nasopharyngeal carcinoma: preliminary results from a phase II clinical trial. Oncotarget. 2017; 8:24572465. https://doi.org/10.18632/oncotarget.13899. 